\title{
Theory and Experiment of a Single-Mode Diode Laser Subject to External Light Injection from Several Lasers
}

\author{
Joerg Troger, Luc Thévenaz, Pierre-Alain Nicati, and Philippe A. Robert
}

\begin{abstract}
A theoretical and experimental study of a gradedindex separate confinement heterostructure (GRIN-SCH) distributed feedback (DFB) multiquantum-well (MQW) diode laser emitting at $1.55 \mu \mathrm{m}$ subject to external light injection from several lasers is presented. Lang's model for the classical single masterslave configuration is extended to include light injection from several master lasers. Free carrier transport effects are taken into account. An experimental validation of the model for two master lasers is made by means of a quantitative comparison between measured and calculated optical spectra. A fiber optics experimental setup makes it possible to measure precisely the power which is injected into the slave laser from each master laser. Measurements and model are in good quantitative agreement.
\end{abstract}

Index Terms-Distributed feedback (DFB) lasers, injection locked oscillators, modeling, optical fibers, quantum-well lasers, spectral analysis.

\section{INTRODUCTION}

$\mathbf{I}^{\mathrm{N}}$ $\mathrm{N}$ OPTICAL frequency division multiplexing networks using coherent detection [1], several lasers may operate at closely spaced frequencies (separated typically by some $\mathrm{GHz}$ ). To avoid undesired optical coupling effects (passive feedback and injection locking) between the different lasers, they need to be isolated. However, it has been pointed out in many papers [2]-[11] that passive feedback and injection locking effects can rise even at very low coupling. Typically, for injection locking phenomena in one laser to occur, only some $0.001 \%$ of its own output power need to be injected into it by another laser. Hence, even if the lasers in a coherent optical network are almost perfectly isolated optically, there is still the possibility for some residual light to pass through the isolators back to the emitters and therefore, to give rise to feedback and injection locking effects between the different light sources. Unbalanced couplers and circulators may lead to asymmetric coupling between the individual lasers and thus to injection-locking like situations.

This paper is devoted to both a theoretical and experimental analysis of a slave laser which is subject to light injection from an arbitrary number of master lasers.

In the theoretical part of this paper, Lang's rate-equation based model [3] used in the classical single master-slave

Manuscript received June 29, 1998; revised December 21, 1998.

The authors are with the Laboratory of Metrology (MET), Swiss Federal Institute of Technology of Lausanne (EPFL), Lausanne CH-1015 Switzerland.

Publisher Item Identifier S 0733-8724(99)02692-4. injection-locking configuration is extended to take account of light injection from an arbitrary number of master lasers. Moreover, the usual rate equation describing the interaction between the carriers and the optical field in bulk semiconductor lasers is adapted to include carrier transport effects in separate confinement heterostructure $(\mathrm{SCH})$ quantum-well diode lasers [12].

In the experimental setup used in this work, the light from the master lasers is launched into a single-mode optical fiber before being injected into the cavity of the slave laser. By using this approach, it is easy to determine accurately the optical power which is coupled from the master lasers into the slave laser. The slave laser is a MQW DFB device emitting at $1.55 \mu \mathrm{m}$ with a $\mathrm{SCH}$ for an improved photon confinement. Frequency detuning and injected power from the master lasers are varied. The steady-state dynamic behavior of the slave laser is studied spectrally with the help of a Fabry-Perot scanning interferometer.

In a further section, theory and experiment are related analytically. The model for two master lasers is validated experimentally by means of spectral analysis. Measured optical spectra are compared with the results from the model. Typical situations including four-wave mixing (FWM), relaxation oscillations, and chaotic behavior are discussed. The slave laser shows some very interesting dynamic behaviors different from those observed with a single master laser.

This paper is organized as follows. Section II introduces the model which takes account of light injection from several master lasers. Section III proceeds with a description of the fiber optics setup used for the experimental work. In Section IV, theory and experiment are related analytically and compared quantitatively by spectral analysis. Section $\mathrm{V}$ is devoted to the conclusion.

\section{MODEL}

In this section, we expound the model describing a singlemode semiconductor laser which is exposed to light injection from several master lasers. The model for two master lasers is presented explicitly at the end of this section. It is validated experimentally in Section IV.

The model is an extension of Lang's approach [3] for the classical single master-slave configuration to include light injection from an arbitrary number of master lasers. The rate equation for the total complex electric field in the slave laser 
cavity can be written as

$$
\begin{aligned}
\frac{d \varepsilon}{d t}= & \left\{-i \omega_{t h}+\frac{1}{2}(G(t)-\gamma)\left(1-i \beta_{c}\right)\right\} \varepsilon(t) \\
& +\frac{1}{\tau_{L}} \sum_{m=1}^{l} \varepsilon_{m}(t)
\end{aligned}
$$

where $\varepsilon_{m}(t)$ is the complex electric field of master laser $m$, also inside the cavity of the slave laser. $\omega_{t h}$ is the angular frequency of the free-running slave laser at threshold. $G(t)$ is the optical gain. An analytical expression for $G(t)$ is given below. $\gamma=\nu_{g}\left(\alpha+\alpha_{i n t}\right)$ is the total photon loss with $\nu_{g}$ the group velocity, $\alpha$ the slave cavity loss, and $\alpha_{i n t}$ the internal loss. $\beta_{c}$ is the linewidth enhancement factor, $\tau_{L}=2 n_{g} L / c$ the cavity round-trip time with $c$ the vacuum light velocity. $n_{g}=c / \nu_{g}$ is the group refractive index and $L$ the slave laser cavity length.

The total intracavity complex electric field $\varepsilon(t)$ and the component $\varepsilon_{m}(t)$ due to master laser $m$ are given by

$$
\begin{aligned}
\varepsilon(t) & =E(t) e^{-i\left(\omega_{R} t+\phi(t)\right)}, \\
\varepsilon_{m}(t) & =E_{m}(t) e^{-i\left(\omega_{R} t+\phi_{m}(t)\right)} .
\end{aligned}
$$

$E(t)$ and $E_{m}(t)$ are the slowly varying field amplitudes. The phase $\phi_{m}(t)=\phi_{m 0}+\left(\omega_{m}-\omega_{R}\right) t$ includes a constant phase term and a time-dependent term taking accounts of the frequency detuning between master laser $m$ and the constant reference. It is evident that this term can be different for all $l$ master lasers.

Putting (2) and (3) into (1) and separating real and imaginary parts, (1) splits into the two following equations:

$$
\begin{aligned}
\frac{d E}{d t}= & \frac{1}{2}(G-\gamma) E+\frac{1}{\tau_{L}} \sum_{m=1}^{l} E_{m} \cos \left(\phi_{m}-\phi\right) \\
\frac{d \phi}{d t}= & -\left(\omega_{R}-\omega_{t h}\right)+\frac{1}{2} \beta_{c}(G-\gamma) \\
& +\frac{1}{\tau_{L}} \sum_{m=1}^{l} \frac{E_{m}}{E} \sin \left(\phi_{m}-\phi\right)
\end{aligned}
$$

where the explicit time dependence of $E, E_{m}, \phi, \phi_{m}$, and $G$ has been dropped for simplicity. It is convenient to express (4) and (5) in terms of the intracavity slave laser photon number $P$ and the injected photon number $P_{m}$. To this end, the field amplitudes $E$ and $E_{m}(m=1 \cdots l)$ are normalized in such a way that $P=V_{p} E^{2}$ and $P_{m}=V_{p} E_{m}^{2}, V_{p}=V / \Gamma$ being the volume of the optical mode, $V$ the active volume, and $\Gamma$ the optical mode confinement factor. Equations (4) and (5) then yield

$$
\begin{aligned}
\frac{d P}{d t}= & (G-\gamma) P+\frac{2}{\tau_{L}} \sum_{m=1}^{l} \sqrt{P P_{m}} \cos \left(\phi_{m}-\phi\right)+R_{s p} \\
\frac{d \phi}{d t}= & -\left(\omega_{R}-\omega_{t h}\right)+\frac{1}{2} \beta_{c}(G-\gamma) \\
& +\frac{1}{\tau_{L}} \sum_{m=1}^{l} \sqrt{\frac{P_{m}}{P}} \sin \left(\phi_{m}-\phi\right) .
\end{aligned}
$$

In (6), the spontaneous emission rate $R_{s p}$ has been added manually. It can be modeled by $R_{s p}=n_{s p} G$, where $n_{s p}$ is the Fermi inversion factor [13].

In case of several master lasers, it is natural to set the reference frequency equal to the free-running slave frequency, i.e., $\omega_{R}=\omega_{t h}$. When dealing with two master lasers, which is the case studied experimentally in Sections III and IV, the equations become

$$
\begin{aligned}
\frac{d P}{d t}= & (G-\gamma) P \\
& +\frac{2}{\tau_{L}}\left\{\sqrt{P P_{1}} \cos \left(\phi_{1}-\phi\right)+\sqrt{P P_{2}} \cos \left(\phi_{2}-\phi\right)\right\} \\
& +R_{s p} \\
\frac{d \phi}{d t}= & \frac{1}{2} \beta_{c}(G-\gamma) \\
& +\frac{1}{\tau_{L}}\left\{\sqrt{\frac{P_{1}}{P}} \sin \left(\phi_{1}-\phi\right)+\sqrt{\frac{P_{2}}{P}} \sin \left(\phi_{2}-\phi\right)\right\} .
\end{aligned}
$$

In the experiment, we use a single-mode MQW DFB diode laser with a graded-index separate confinement heterostructure (GRIN-SCH). Two rate equations model the carrier transport from the barrier region ( $\mathrm{SCH}$ ) into the active layers (QW's) and vice versa and their interaction with the photon population [12]. They read

$$
\begin{aligned}
\frac{d N_{B}}{d t} & =\eta_{i} \frac{I}{q}-\gamma_{B Q} N_{B}+\gamma_{Q B} N \\
\frac{d N}{d t} & =\gamma_{B Q} N_{B}-\left(\gamma_{e}+\gamma_{Q B}\right) N-G P
\end{aligned}
$$

where $N_{B}$ and $N$ are the carrier number in the barrier region and in the active region, respectively. $I$ is the slave laser drive current, $\eta_{i}$ the internal quantum efficiency, and $q$ the unit charge. The nonlinear gain is given by $G=$ $\left(\Gamma g_{0} \nu_{g}\right) /\left(1+P / P_{s}\right) \log \left\{\left(N+N_{s}\right) /\left(N_{0}+N_{s}\right)\right\}$ [12], [13]. In this expression, $g_{0}$ is a linear gain coefficient and $N_{s}$ a parameter of the logarithmic gain with $N_{s}=n_{s} V . N_{0}$ is the active layer carrier number at transparency related to its carrier density $n_{0}$ by $N_{0}=n_{0} V . P_{s}$ is the saturation photon number. The other parameters of the gain have been defined before. $\gamma_{e}=A_{n r}+B(N / V)+C(N / V)^{2}$ is the total carrier loss in the active region, with $A_{n r}$ the nonradiative recombination rate, $B$ the radiative recombination coefficient taking into account the carrier loss induced by spontaneous emission, and $C$ the Auger recombination coefficient. $\gamma_{B Q} N_{B}$ is the loss rate of carriers from the $\mathrm{SCH}$ region to the quantum wells, whereas $\gamma_{Q B} N$ describes the rate at which the carriers escape from the active region into the $\mathrm{SCH}$ layer.

Using (10) and (11) instead of the usual single rate equation for the carriers [12] and [13]

$$
\frac{d N}{d t}=\eta_{i} \frac{I}{q}-\gamma_{e} N-G P
$$

changes the dynamics of the interaction between the laser field and the carriers but does not affect the steady-state values of $P$ and $N$. This can be seen easily by putting (10) in (11) 
TABLE I

Slave Laser Parameter Values Used in the Computer Simulations

\begin{tabular}{|c|c|c|c|}
\hline Parameter & Symbol & Value & Unit \\
\hline Cavity length* & $L$ & 1200 & $\mu \mathrm{m}$ \\
\hline Active-region width* & $w$ & 1.5 & $\mu \mathrm{m}$ \\
\hline Active-region thickness* & $d$ & 0.028 & $\mu \mathrm{m}$ \\
\hline Mode confinement factor* & $\Gamma$ & 0.045 & \\
\hline Group refractive index* & $n_{g}$ & 3.6 & \\
\hline Cavity photon loss & $\alpha$ & 11.5 & $\mathrm{~cm}^{-1}$ \\
\hline Internal photon loss* & $\alpha_{i n t}$ & 20 & $\mathrm{~cm}^{-1}$ \\
\hline Linear gain coefficient & $g_{0}$ & 2700 & $\mathrm{~cm}^{-1}$ \\
\hline Logarithmic gain parameter & $n_{s}$ & $0.1 \cdot 10^{18}$ & $\mathrm{~cm}^{-3}$ \\
\hline Carrier density at transparency & $n_{0}$ & $2.1 \cdot 10^{18}$ & $\mathrm{~cm}^{-3}$ \\
\hline Nonradiative recombination rate* & $A_{n r}$ & $2.5 \cdot 10^{8}$ & $\mathrm{~s}^{-1}$ \\
\hline Radiative recombination coefficient* & $B$ & $1.0 \cdot 10^{-10}$ & $\mathrm{~cm}^{3} / \mathrm{s}$ \\
\hline Auger recombination coefficient ${ }^{*}$ & $C$ & $5.0 \cdot 10^{-29}$ & $\mathrm{~cm}^{6} / \mathrm{s}$ \\
\hline Linewidth enhancement factor* & $\beta_{c}$ & 3 & \\
\hline Drive current & $I$ & 50,100 & $\mathrm{~mA}$ \\
\hline Fermi inversion factor & $n_{s p}$ & 1.6 & \\
\hline Internal quantum efficiency & $\eta_{i}$ & 0.8 & \\
\hline Saturation photon number* & $P_{s}$ & $2.2 \cdot 10^{7}$ & \\
\hline SCH to QW carrier transport coeff. & $\gamma_{B Q}$ & $2.5 \cdot 10^{10}$ & $\mathrm{~s}^{-1}$ \\
\hline QW to SCH carrier transport coeff. & $\gamma_{Q B}$ & $5.0 \cdot 10^{9}$ & $\mathrm{~s}^{-1}$ \\
\hline
\end{tabular}

presuming steady state for the barrier carrier number, i.e., $d N_{B} / d t=0$.

A linear stability analysis of the steady-state solutions in the single master-slave injection-locking configuration [2]-[8] described by (6) and (7) (put $l=1$ ) in combination with (10) and (11) to include carrier transport effects, yields four eigenvalues, two of which are purely real and the other two are complex conjugated in the static locking range. We observe an enhanced damping of the relaxation oscillations compared to the case where carrier transport is neglected [i.e., when using (12) instead of (10) and (11)]. Thereby, the inclusion of carrier transport effects results in a shift of the edges of undamped relaxation oscillations and chaos toward higher injections. The dynamic response of the slave laser being altered by the carrier transport in the single master-slave configuration, carrier transport effects are also included in modeling the $\mathrm{SCH}$ slave laser exposed to light injection from several master lasers.

The parameter values of the slave laser used in the computer simulations are listed in Table I. Those with an asterisk were provided directly by the laser manufacturer [14]. The other parameter values were chosen in their typical ranges [12], [13] and for the model to best fit the experimental light-

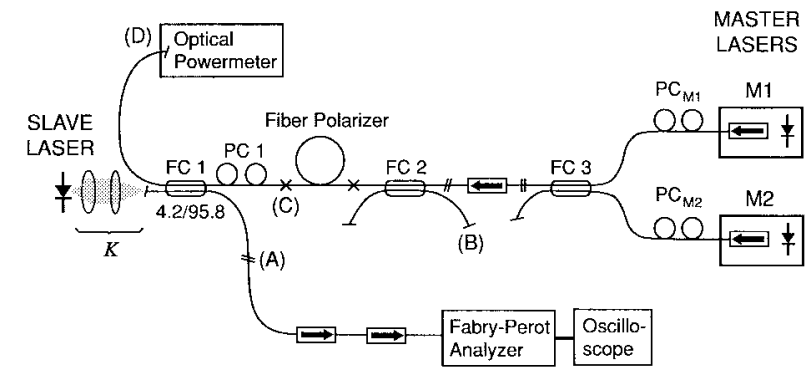

Fig. 1. Experimental setup showing the fiber-coupled master and slave lasers. FC1, FC2, and $\mathrm{FC} 3$ are broad-band fiber couplers; $\mathrm{PC} 1, \mathrm{PC}_{\mathrm{M} 1}$, and $\mathrm{PC}_{\mathrm{M} 2}$ polarization controllers.

current characteristic (threshold and slope) and the relaxation oscillation frequency. The relaxation oscillation frequency at a given bias current was determined by some low power injection locking measurements with a single master laser turned on. It must be indicated that $L$ is the overall cavity length of the three section laser and that the active-region thickness $d$ comprises the four quantum wells, each $7 \mathrm{~nm}$ thick (cf. Section III).

\section{EXPERIMENTAL SETUP With Two MASTER LASERS}

The single-mode fiber optics setup used in our experiment is shown in Fig. 1. The two masters M1 and M2 are $1.55 \mu \mathrm{m}$ DFB lasers. The slave is a three-section GRIN-SCH MQW DFB diode laser with four quantum wells and AR coated facets. To ensure uniform pumping of the three sections [14], the latter are driven by a single current supply. At room temperature, the slave laser emits $P_{\text {out }}^{f r}=8.8 \mathrm{~mW}$ at a bias current of $I=100 \mathrm{~mA}$. The threshold current is $25 \mathrm{~mA}$. All the lasers are frequency stabilized using thermoelectric coolers. Frequency tuning between the master lasers and the slave laser is achieved by varying the bias current and the operating temperature of the master lasers.

The two master laser diodes are isolated by $70 \mathrm{~dB}$ from the slave laser in order to prevent the radiation emitted by the slave laser from being injected into the master lasers. Angled fiber ends help minimize losses and reflections. The polarizing fiber has a measured extinction ratio of $41 \mathrm{~dB}$. A Fabry-Perot scanning interferometer $(\mathrm{FSR}=10-55 \mathrm{GHz}$ depending on measurement, finesse $=1000$ ) is used to study spectrally the behavior of the slave laser under light injection from the two master lasers.

The ratio between the crosscoupled and the uncoupled power in the fiber coupler $\mathrm{FC} 1$ is written as $(1-\eta) / \eta$, with a measured $\eta=0.958$. Loss in the coupler is negligible.

The factor $K$ in Fig. 1 stands for the light coupling efficiency between the slave laser and the optical fiber. It consists of the losses due to the mismatch between the optical modes in the slave laser waveguide and in the fiber, the Fresnel losses at the lenses and at the angled fiber interface. By reciprocity, $K$ also describes the coupling of light between the fiber and the cavity of the slave laser. It can be determined by

$$
K=\frac{P_{(A)} / \eta}{P_{\text {out }}^{f r}}
$$


with $P_{(A)}$ the optical power measured at point (A), where the fiber connectors are temporarily opened. In our configuration, typical values for $K$ are near 0.3 with good alignment of the optical elements.

We define $P_{\mathrm{inj}}^{(1)}$ and $P_{\mathrm{inj}}^{(2)}$ as the fractions of light from master lasers M1 and M2 at the interface of the slave matching the mode of the slave laser waveguide. For master laser M1, we can write

$$
P_{\mathrm{inj}}^{(1)}=\frac{1-\eta}{\eta} K P_{(D)}^{(1)}
$$

where $P_{(D)}^{(1)}$ is the optical power measured at point (D) with master laser M1 turned on and M2 turned off. An analogous expression is found for $P_{\mathrm{inj}}^{(2)}$.

In this paper, we limit our experimental investigations to two master lasers. However, it is obvious that the setup can be easily extended for an arbitrary number $l$ of master lasers. The extra master lasers have then to be connected to the setup via additional fiber couplers. With the present fiber optics setup, the coupling factor $K$ given by (13) is the same for every additional master laser. As it is shown at the beginning of Section IV, $P_{\mathrm{inj}}^{(m)}(m=1 \cdots l)$ given by (14) is related to the injected intracavity photon number $P_{m}$ by a linear relationship involving the slave laser cavity loss. Measurements and model can therefore be fully related analytically.

Conversely, in free-space systems, where no optical fiber is used, the spatial overlap between the optical mode in free space and in the slave laser waveguide differs for each master laser. For this reason, the coupling factors between the individual master lasers and the slave laser are all unequal and have to be determined separately. This makes free-space setups quite inappropriate for injection-locking experiments with several master lasers.

Special attention has to be paid to the polarization of the injected fields. Intracavity and injected fields have to be polarized identically in order to maximize their interaction and to avoid polarization switching between transverse electric (TE) and transverse magnetic (TM) modes. Polarization matching is achieved as follows: output (B) monitors the part of the slave power which is transmitted through the fiber polarizer. The power transmission is maximized with the polarization controller PC 1. By doing so, the slave laser light, which is linearly polarized at the fiber interface, has the same polarization state at the input $(\mathrm{C})$ of the polarizer. In the absence of nonreciprocal effects [15], the light generated by any master laser is then also linearly polarized at the input facet of the slave.

The exact amount of master laser light $P_{\mathrm{inj}}^{(1)}$ and $P_{\mathrm{inj}}^{(2)}$ at the interface of the slave matching the mode of the slave laser waveguide is adjusted with the polarization controller PCM1 and PCM2, respectively.

\section{VAlidation OF THE MOdEL FOR Two MASTER LASERS}

Section II has introduced a model for a single-mode slave laser which is subjected to light injection from an arbitrary number of master lasers. In the current section, the model for two master lasers is validated experimentally. For this purpose, some measured power spectra are compared with the calculated results from the model.

For a quantitative comparison to be possible, theory and experiment have first to be linked. Model and experiment can be related analytically via the slave laser cavity loss $\alpha$. In fact, the injected energy per round-trip time from the master laser $m$ into the slave laser may be written as

$$
\frac{\hbar \omega_{t h} P_{m}}{\tau_{L}}=P_{\mathrm{inj}}^{(m)} 2 \alpha L
$$

from which

$$
P_{m}=\frac{2 \tau_{L} \alpha L}{\hbar \omega_{t h}} P_{\mathrm{inj}}^{(m)} .
$$

Moreover, the total slave intracavity photon number $P$ and the outcoupled optical power per facet $P_{\text {out }}$ are related by the usual equation [13]

$$
P=\frac{\tau_{L}}{\hbar \omega_{t h} \alpha L} P_{\text {out }}
$$

Here, the cavity loss $\alpha$ only encompasses the distributed feedback loss of the slave laser, since its facets are AR coated. Together with (14) linking $P_{\mathrm{inj}}^{(m)}$ to the directly measurable power $P(D)$, (16) and (17) provide the necessary connection between theory and experiment.

Equations (8)-(11) of Section II describe a single-mode slave laser in which light from two master lasers is injected. In the remaining part of this section, we validate this model experimentally using the setup of Section III. The injected power from the two master lasers and their frequency detunings with respect to the free-running slave laser are varied over a certain range. This is shown to give rise to different interesting dynamic behaviors of the slave laser.

The verification of the model for two master lasers is based on a quantitative comparison between several measured and calculated power spectra. The theoretical power spectra were calculated by taking the fast Fourier transform (FFT) of the steady-state slave intracavity electric field over a given time window. Experimentally, the power spectra were determined using the Fabry-Perot analyzer. The spectra were normalized with respect to the power of the free-running slave laser.

All in all, more than 40 spectra were measured and compared with the results given by the model. In most situations, the slave laser was biased at $100 \mathrm{~mA}$, i.e., four times the threshold current. However, some comparisons were done with the slave laser biased at $50 \mathrm{~mA}$. The power from the two master lasers $(m=1,2)$ indicated by $P_{\text {inj }}^{(m)} / P_{\text {out }}^{f r}$ varied from $1.0 \cdot 10^{-4}$ to $1.5 \cdot 10^{-2}$ for detunings $\Delta v_{m}=\left(\omega_{m}-\omega_{t h}\right) /(2 \pi)$ between approximately -10 and $+10 \mathrm{GHz}$.

In about $50 \%$ of all situations, measurements and model give quasi-identical spectra. The spectral peaks agree very well both in position and amplitude. Another $30 \%$ give qualitatively similar measured and calculated spectra, but with somewhat inconsistent peak amplitudes and/or peak positions. Finally, in about $20 \%$ of the comparisons, measurement and model disagree completely. 
Possible reasons for inconsistencies include different operating points in measurement and model, omitted phase noise in the model, neglected geometry of the three section slave laser (three sections are lumped together in one section) and imprecise parameter values. Particularly, in the model, the peaks have zero linewidth due to the neglected phase noise. Experimentally, however, the different linewidths of the master lasers, the free-running slave laser and the observed conjugated signals due to nearly degenerate four-wave mixing (NDFWM) have an impact on the relative peak strengths of the normalized power spectra. Finally, in more than half of the inconsistent cases, the slave laser was biased at $50 \mathrm{~mA}$. In these situations, a disagreement between theory and experiment is mainly ascribed to the mode hop of the real slave laser occurring at drive currents between 35 and $40 \mathrm{~mA}$, which the model does not take into account. When biased at currents near the mode hop, the slave laser exhibits somewhat untypical behaviors in the single master-slave configuration. It is reasonable to assume that its behavior may also be modified when exposed to light injection from two lasers.

Experimentally, we observed four different categories of spectra, which are illustrated in the examples Fig. 2(a)-(d). We presume that other behaviors may occur depending on injection level and detuning. Particularly, conditions of period doubling similar to those with a single master laser, could possibly be found. In Fig. 2, the measured spectra are in the left column and the corresponding calculated spectra in the right column. The horizontal axis denotes the frequency shift between the master and the free-running slave (i.e., the freerunning slave laser frequency is the reference), whereas the vertical axis indicates the normalized spectral density on a linear scale.

The situation in Fig. 2(a) illustrates the first class of typical behavior. The slave laser is injection locked to the first master laser $(\mathrm{M} 1=\mathrm{S})$. Nearly degenerate four-wave mixing (NDFWM) [16], [17] of different waves can be made responsible for the fields $C_{i}$, well visible in the measured and the calculated spectra. NDFWM in an injection-locked semiconductor laser was studied in detail by Li and Petermann in [18]. NDFWM between the injection-locked slave and the master M2 accounts for the conjugate signals $\mathrm{C} 1$ and $\mathrm{C} 2$. NDFWM between the injection-locked slave and the master M2 accounts for the conjugate signals $\mathrm{C} 1$ and $\mathrm{C} 2$. The other waves can be explained by a kind of cascade NDFWM [19], where one of the involved waves is itself generated by NDFWM. For instance, C3 may be produced by $\mathrm{M} 1=\mathrm{S}$ and $\mathrm{C} 1$ or, alternatively, by $\mathrm{C} 2$ in combination with $\mathrm{M} 1=\mathrm{S}$. Wave $\mathrm{C} 4$, in turn, is created by M2 mixing with $\mathrm{C} 1$. Additionally, $\mathrm{C} 4$ possibly stems from the mixing of $\mathrm{C} 1$ with $\mathrm{C} 3$, where both waves themselves result from NDFWM. However, we assume this contribution to be negligible, due to the very weak amplitude of $\mathrm{C} 3$, which appears squared in the mixing term.

In Fig. 2(b), which is an example of the second category of characteristic spectra, theory and experiment give very similar results. A closer look at the magnified calculated spectrum reveals a large number of different peaks, though most of them are very weak in comparison with the main peaks. Some of the low power peaks visible in the calculated spectrum [e.g., peak Fig. 2(d)] are imperceptible in the measurement, as they are hidden in the background noise of the detection system. In theory, the regenerative amplification of master M1 is somewhat more efficient than in reality. The opposite is true for M2. The slave is locked on neither of the two master lasers. The slave and the two master lasers form a set of base frequencies [20], [21]. Let $\omega_{s}, \omega_{1}$, and $\omega_{2}$ be the (angular) optical frequencies of the slave laser $S$ and the master lasers M1 and M2, respectively. Mathematically, the frequencies of the other signals are each determined by a linear combination of the base frequencies. Physically, these signals are all generated by wave mixing in the nonlinear slave laser medium. For instance, NDFWM between M1 and the slave S generates the upconverted conjugate signal (a) by $\omega_{a}=2 \omega_{s}-\omega_{1}$. The same physical phenomenon is responsible for peak (b), which is the downconverted conjugate signal of M2 and the slave laser, i.e., $\omega_{b}=2 \omega_{s}-\omega_{2}$. Furthermore, NDFWM creates the waves (c) and (d) with angular frequencies $\omega_{c}=\omega_{s}+\omega_{1}-\omega_{2}$ and $\omega_{d}=\omega_{s}-\omega_{1}+\omega_{2}$, respectively. Wave (e) may be interpreted as resulting from cascade NDFWM [19] between the slave laser S, the master laser M1, and the wave (b), which itself is generated by NDFWM. In this perspective, it is written as $\omega_{e}=\omega_{s}-\omega_{1}+\omega_{b}$. Alternatively, in terms of the three base frequencies, wave (e) reads $\omega_{e}=3 \omega_{s}-\omega_{1}-\omega_{2}$. Equations (8)-(11) yield a degree 3 quasiperiodic final motion [20].

In the situation of Fig. 2(c), contrary to Fig. 2(a) and (b), the slave laser is biased at $50 \mathrm{~mA}$. Higher injection ratios are now accessible. In Fig. 2(c), measured and calculated spectra are fully consistent. In this group of spectra, the slave laser typically exhibits undamped relaxation oscillations (indicated by R), similar to those observed in the single master-slave configuration. In addition to the relaxation oscillation sidebands, the spectra contain the two regeneratively amplified peaks of the injected signals M1 and M2 from the master lasers. Fig. 2(c) is a particularly interesting example of the class of undamped relaxation oscillations. The frequency difference between the two master lasers equals the relaxation oscillation frequency of the slave laser. In the measurement, we first injection-locked the slave laser on M1 and then tuned M2 to the first relaxation oscillation sideband at the lower frequency side. This made it possible to get the very interesting result experimentally. More generally, for such a behavior to occur, the frequency difference between the two master lasers must be a multiple of the slave relaxation oscillation frequency. The slave laser power is shared between the master lasers. The steady-state solution of (8)-(11) in case Fig. 2(c) is periodic with a period equal to the relaxation oscillation frequency.

The broad-band spectra of case Fig. 2(d) indicate a chaotic behavior, which is the fourth category of common spectra. In the displayed example, measurement and theory agree qualitatively. Both spectra clearly show the regeneratively amplified injected signals M1 and M2. However, the relative amplitudes of M1 and M2 are considerably different in theory and experiment. The energy is widely spread between M1 and M2 near the frequency of the free-running slave laser, which itself is somewhat lowered by the light injection [3]. In this region, theory and experiment agree well. Numerical steady-state solutions of (8)-(11) yield a chaotic attractor. As 
Measurement

$$
\begin{aligned}
I & =100 \mathrm{~mA} \\
P_{i n j}^{(1)} / P_{\text {out }}^{f f} & =2.31 \cdot 10^{-3} \\
\Delta v_{1} & =0.46 \mathrm{GHz} \\
P_{\text {inj }}^{(2)} / P_{\text {out }}^{f f} & =2.07 \cdot 10^{-3} \\
\Delta v_{2} & =0.94 \mathrm{GHz}
\end{aligned}
$$

$$
\begin{aligned}
I & =100 \mathrm{~mA} \\
P_{\text {inj }}^{(1)} / P_{\text {out }}^{f r} & =1.45 \cdot 10^{-3} \\
\Delta \mathrm{v}_{1} & =-1.21 \mathrm{GHz} \\
P_{\text {inj }}^{(2)} / P_{\text {out }}^{f r} & =1.55 \cdot 10^{-3} \\
\Delta \mathrm{v}_{2} & =1.39 \mathrm{GHz}
\end{aligned}
$$

$I=50 \mathrm{~mA}$

$P_{\text {inj }}^{(1)} / P_{\text {out }}^{f r}=5.13 \cdot 10^{-3}$

$\Delta v_{1}=0.75 \mathrm{GHz}$

$P_{i n j}^{(2)} / P_{o u t}^{f f}=3.35 \cdot 10^{-3}$

$\Delta \mathrm{v}_{2}=-3.13 \mathrm{GHz}$
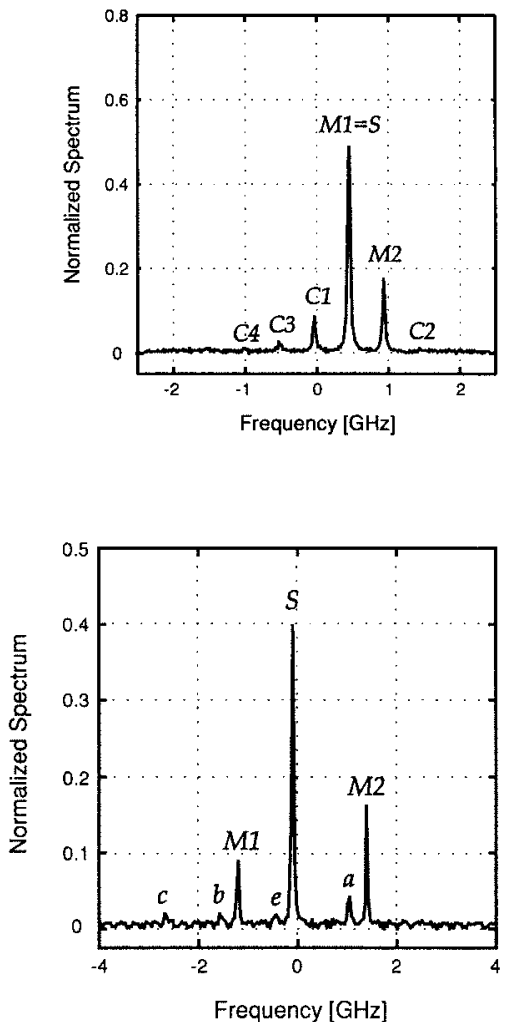

(b)
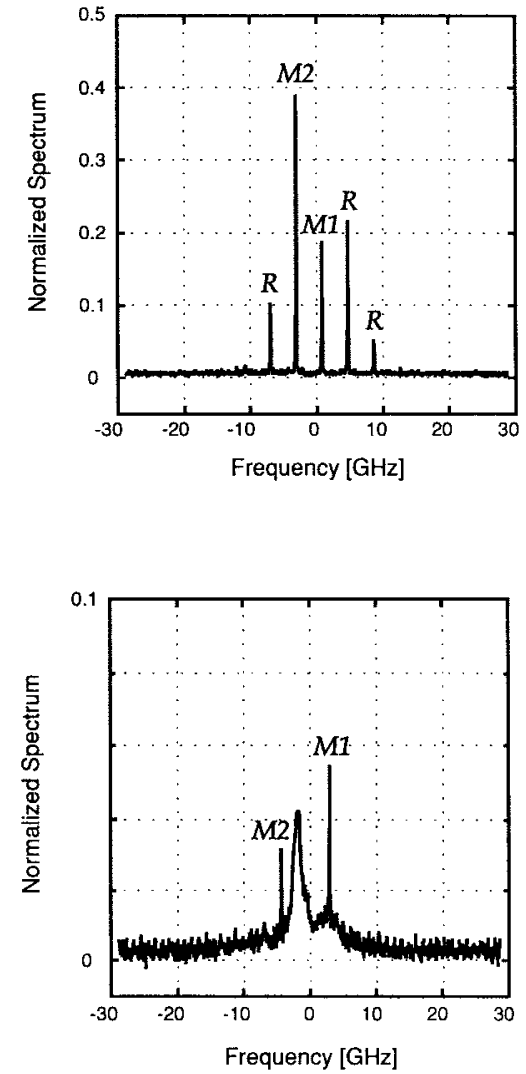

(c)

Model

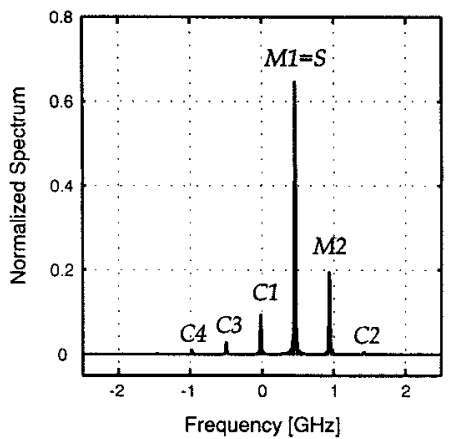

(a)
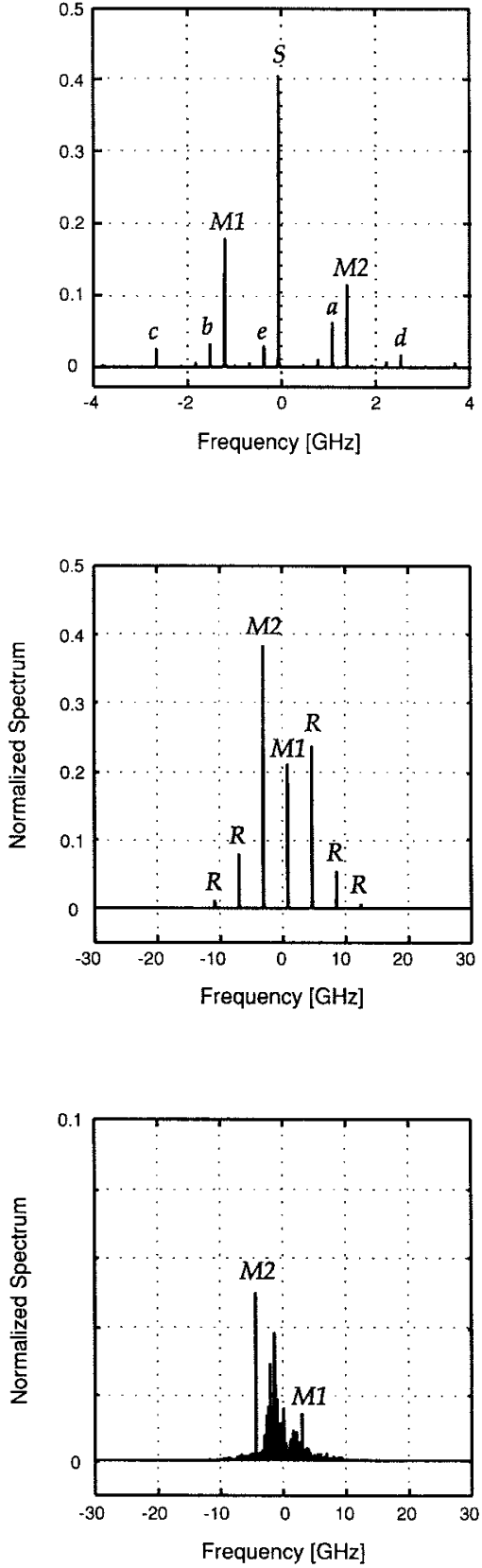

(d)

Fig. 2. Measured and calculated power spectra at different operating points. $\mathrm{M} 1=$ first master laser, $\mathrm{M} 2=$ second master laser, $\mathrm{S}=$ slave laser, $\mathrm{R}=$ relaxation oscillation sidebands, $C_{i}, a, b, c, d, e=$ signals generated by FWM. 
mentioned earlier, the main reason for the partial inconsistency between theory and experiment in the case of Fig. 2(d) is ascribed to a mode jump of the slave laser occurring at low drive currents.

\section{CONCLUSIONS}

In this paper, we have presented a theoretical and experimental study of a single-mode diode laser subject to external light injection from several lasers. The work may be relevant in coherent optical networks, where the different lasers with closely spaced wavelengths may interact despite optical isolation.

The model is an extension of Lang's model [3] used for the classical single master-slave configuration to include light injection from several master lasers. The slave is a single-mode MQW diode laser with an $\mathrm{SCH}$. Free carrier transport between the barrier and the active regions is taken into account. It turns out that such carrier transport effects are responsible for the modified dynamic response of the semiconductor laser not only in free-running [12] but also in light injection conditions. More seriously, the inclusion of the carrier transport effects results in an enhanced damping of relaxation oscillations, and thereby, in the single master-slave configuration, in a shift of the boundary of undamped relaxation oscillations to higher injections.

The fiber optics setup used in the experiment has many advantages compared to traditional free-space systems. The most significant benefit arises from the fact that it is easily possible to determine precisely the exact power which is injected into the slave from each master laser. The coupling of the optical power injected from the fiber into the slave laser is the same for all the master lasers. The somewhat troublesome calibration procedures required for every individual master laser in free-space systems are no longer needed. This makes a quantitative comparison between model and measurement easily possible.

The model describing a single-mode slave diode laser under light injection from two master lasers is verified experimentally. The comparison between the theory and the experiment is based on spectral analysis. Experimentally, four classes of typical behavior have been found. NDFWM can be observed between one master and the slave laser, which is injection locked to the other master laser. This situation has been studied in detail in [18]. NDFWM also occurs between the two master lasers and the unlocked slave laser. Very interesting cascade NDFWM effects [19] have also been identified. At stronger injection, we observe situations of undamped relaxation oscillations, similar to those observed in the classical single master-slave experiment [2]-[8], [11]. Finally, at even higher injection, chaotic behavior can be observed in some cases.

Model and measurement agree very well in more than 50\% and reasonably well for another $30 \%$ of the studied cases. The reasons for the remaining inconsistent $20 \%$ are manifold. They include different operating points in measurement and model, omitted phase noise in the model, neglected geometry of the three section slave laser and inaccurate parameter values.
Furthermore, mode hopping of the real slave laser at low drive currents may be the most significant cause for inconsistency between theory and experiment.

By means of additional fiber couplers, an arbitrary number of master lasers can be linked to the experimental setup. Constraints are related with the availability of master diode lasers, whose frequencies must lie closely enough to each other. Nowadays, in the era of wavelength division multiplexing optical networks, this is actually no longer a problem. It should then be possible to validate experimentally the model of Section II for an arbitrary number of master lasers.

\section{REFERENCES}

[1] G.-D. Khoe, "Coherent multicarrier lightwave technology for flexible capacity networks," IEEE Commun. Mag., pp. 22-33, Mar. 1994.

[2] S. Kobayashi and T. Kimura, "Injection locking in AlGaAs semiconductor laser," IEEE J. Quantum Electron., vol. 17, pp. 681-689, May 1981.

[3] R. Lang, "Injection locking properties of a semiconductor laser," IEEE J. Quantum Electron., vol. 18, no. 6, pp. 976-983, 1982.

[4] C. H. Henry, N. A. Olsson, and N. K. Dutta, "Locking range and stability of injection locked $1.54 \mu \mathrm{m}$ InGaAsP semiconductor lasers," IEEE $J$. Quantum Electron., vol. 21, pp. 1152-1156, Aug. 1985.

[5] F. Mogensen, H. Olesen, and G. Jacobsen, "Locking conditions and stability properties for a semiconductor laser with external light injection," IEEE J. Quantum Electron., vol. 21, pp. 784-793, July 1985.

[6] R. Hui, A. D'Ottavi, A. Mecozzi, and P. Spano, "Injection locking in distributed feedback semiconductor lasers," IEEE J. Quantum Electron., vol. 27, pp. 1688-1695, June 1991.

[7] L. Li, "Static and dynamic properties of injection-locked semiconductor lasers," IEEE J. Quantum Electron., vol. 30, pp. 1701-1708, Aug. 1994.

[8] V. Kovanis, A. Gavrielides, T. B. Simpson, and J. M. Liu, "Instabilities and chaos in optically injected semiconductor lasers," Appl. Phys. Lett., vol. 67 , no. 19 , pp. $2780-2782,1995$.

[9] R. Lang and K. Kobayashi, "External optical feedback effects on semiconductor injection laser properties," IEEE J. Quantum Electron., vol. 16 , pp. $347-355$, Mar. 1980

[10] C. H. Henry and R. F. Kazarinov, "Instability of semiconductor lasers due to optical feedback from distant reflectors," IEEE J. Quantum Electron., vol. 22, pp. 294-301, Feb. 1986.

[11] J. Sacher, D. Baums, P. Panknin, W. Elsässer, and E. O. Göbel, "Intensity instabilities of semiconductor lasers under current modulation, external light injection, and delayed feedback," Phys. Rev. A, vol. 45, no. 3, pp. 1893-1905, 1992.

[12] L. A. Coldren and S. W. Corzine, Diode Lasers and Photonic Integrated Circuits. New York: Wiley, 1995.

[13] G. P. Agrawal and N. K. Dutta, Semiconductor Lasers, 2nd ed. New York: Van Nostrand Reinhold, 1993.

[14] S. Nilsson, T. Kjellberg, T. Klinga, R. Schatz, J. Wallin, and K. Streubel, "Improved spectral characteristics of MQW-DFB lasers with incorporation of multiple phase-shifts," IEEE J. Lightwave Technol., vol. 13, pp. 434-441, Mar. 1995.

[15] E. Brinkmeyer, "Forward-backward transmission in birefringent singlemode fibers: Interpretation of polarization-sensitive measurements," Opt. Lett., vol. 6, no. 11, pp. 575-577, 1981.

[16] G. P. Agrawal, "Four-wave mixing and phase conjugation in semiconductor laser media," Opt. Lett., vol. 12, no. 4, pp. 260-262, 1987.

[17] A. Uskov, J. Mork, and J. Mark, "Wave mixing in semiconductor laser amplifiers due to carrier heating and spectral-hole burning," IEEE J. Quantum Electron., vol. 30, pp. 1769-1781, Aug. 1990.

[18] L. Li and K. Petermann, "Small-signal analysis of optical-frequency conversion in an injection-locked semiconductor laser," IEEE J. Quantum Electron., vol. 30, pp. 43-48, Jan. 1994.

[19] R. Nietzke, P. Panknin, W. Elsässer, and E. O. Göbel, "Four-wave mixing in GaAs/AlGaAs semiconductor lasers," IEEE J. Quantum Electron., vol. 25, pp. 1399-1406, June 1989.

[20] J. M. T. Thompson and H. B. Stewart, Nonlinear Dynamics. New York: Wiley, 1986

[21] T. S. Parker and L. O. Chua, "Chaos: A tutorial for engineers," Proc. Inst. Elect. Eng., vol. 75, no. 8, pp. 982-1008, 1987.

[22] B. Tromborg and J. Mork, "Nonlinear injection locking dynamics and the onset of coherence collapse in external cavity lasers," IEEE $J$. Quantum Electron., vol. 26, pp. 642-654, Apr. 1990. 
Joerg Troger received the M.S. degree in microengineering from the Swiss Federal Institute of Technology of Lausanne (EPFL), Switzerland, in 1993. $\mathrm{He}$ is currently working toward the Ph.D. degree.

He then joined the ASCOM Tech Research Departments in Maegenwil and Bern, Switzerland, where he worked as a technical trainee on radio wave propagation and high-speed optoelectronics. Since 1994, he has been with the Metrology Laboratory of the EPFL, where his primary research is on injection locking with semiconductor lasers.

Luc Thévenaz received the B.Sc. degree in astrophysics from the Observatory of Geneva, Switzerland, in 1982 and the Ph.D. degree in physics from the University of Geneva, Switzerland, in 1988.

In 1983, he joined the Group of Applied Physics at the University of Geneva, where he has been engaged in research work on single-mode fiber measurement techniques. In 1988, he joined the Laboratory of Metrology of the Swiss Federal Institute of Technology of Lausanne (EPFL), Switzerland, where is presently a Research Manager and Lecturer. He manages many research projects dealing with guided-wave measurement techniques, Brillouinscattering optical fiber sensors, nonlinearities in fibers, and spectrascopic techniques. In 1991, he visited the University of Rio de Janeiro, Brazil, and Stanford University, Stanford, CA, where he participated in the development of a Brillouin laser fiber gyroscope.
Pierre-Alain Nicati, photograph and biography not available at the time of publication.

Philippe A. Robert received the Diploma degree in physics engineering in 1961 and the Ph.D. degree in 1968 from the Swiss Federal Institute of Technology of Lausanne (EPFL), Switzerland.

From 1968 to 1974, he was with Les Cābleries et Trébleries de Cossonay, where his research interests are in new products and new manufacturing processes, including optical fibers. From 1974 to 1979, he has been engaged in the development and installation of the first optical cables made in Switzerland. Since 1979, he has been a Professor at the EPFL and Head of the Laboratory of Metrology. His current research activities concern guided optics, optical fibers, and integrated optics with applications in optical signal processing, sensors, and telecommunications. 